\title{
A SYSTEMATIC NON-INVASIVE APPROACH FOR REHABILITATION OF TRAUMATIZED MAXILLARY LATERAL INCISOR - A CASE REPORT.
}

\section{Dental Science}

P Karunakar

M.S. Rangareddy

Basa Srinivas
Karteek

\section{Chavva Lakshmi Charan Reddy*}

Gandikota

Akshara
Principal \& HOD, Department of Conservative Dentistry and Endodontics, Panineeya Institute of Dental Sciences and Research Centre, Hyderabad, Telangana, India.

Professor, Department of Conservative dentistry and Endodontics, Panineeya Institute of Dental Sciences and Research Centre, Hyderabad, Telangana, India.

Reader, Department of Conservative dentistry and Endodontics, Panineeya Institute of Dental Sciences and Research Centre, Hyderabad, Telangana, India.

Post Graduate student, Department of Conservative dentistry and Endodontics, Panineeya Institute of Dental Sciences and Research Centre, Hyderabad, Telangana, India. *Corresponding Author

Under Graduate student, Sri Ramachandra Dental College \& Hospital, Porur, Chennai, Tamilnadu, India.

\section{ABSTRACT}

Coronal fractures of permanent anterior teeth by trauma are the most common type of injury. Many factors are considered in the attempt to provide optimal mechanical properties, aesthetic, and longevity. Discomfort, serious psychological, esthetic, functional, and phonetic problems are related with anterior crown fracture, this may also affect social communications and it poses a challenge for the dentist, to save these teeth. The restoration of traumatically injured teeth with substantial loss of coronal structure necessitates endodontic treatment followed by post insertion into canal space so that foundation restoration can be strengthened to receive the crown. In this case report, such case is described with fiber-reinforced post and core system.

\section{KEYWORDS}

Crown fracture, Cast post, Fiber-reinforced post, Porcelain fused to metal crown

\section{INTRODUCTION:}

The most common type of injury in the permanent dentition is seen as fracture of anterior teeth which mostly occur among children and adolescent reported up to $25 \%$ of the total patient population. The incidence of complicated crown fractures ranges from $2 \%$ to $13 \%$ of all dental injuries and the most commonly involved teeth are the maxillary anteriors. ${ }^{(1)}$ Anterior crown fractures lead to discomfort and serious psychological, esthetic, functional, and phonetic problems that can affect social relationships.

In cases where the teeth are severely fractured, endodontic treatment and placement of intracanal posts become compulsory, before crown restoration. An alternative to prefabricated metal posts, cast post for the restoration of endodontically treated teeth have emerged with numerous types of fiber reinforced posts in recent years. ${ }^{(3)}$ The reinforced fiber which is used to construct an intracanal post offers superiorities over other systems, such as relative ease of manipulation, translucency, and resin composite crown reinforcement. ${ }^{(4)}$

The technology evolution has enabled manufacturers today to provide fiber posts that-besides offering superior aesthetic and mechanical properties (which are the first qualities to be appreciated in comparison with metal or cast post) are also radio opaque and available in a great variety of shapes.

This article reports such a case treated with Glass Fiber Reinforced Post and Core for the restoration of traumatically fractured maxillary left permanent lateral incisor.

\section{Case Report:}

A 22 year old female patient came to the Department of Conservative Dentistry and Endodontics, Panineeya Dental College and Research Centre, Hyderabad, with the chief complaint of fractured upper front teeth with a unaesthetic appearance since 6 months after sustaining trauma due to accidental fall. On examination, there was no pain and swelling. Ellis class III fracture was diagnosed during intraoral examination (Fig 1a,1b). Radiolucency involving enamel, dentine, and pulp was seen in radiographic examination (Fig 2). Vitality test was done using Electric pulp test (Waldent Electric Pulp Tester) and recorded as negative response (Non-vital). After discussing various treatment plans with the patient, it was decided to restore the complex crown fracture with fiber post and core followed by Porcelain fused to metal crown. Informed consent was obtained from the patient.
Local Anesthesia was administered using 2\% Lignocaine. Access was gained with no. 2 round bur (Dentsply Maillefer Pvt Ltd) and Endo-Z bur (Dentsply Maillefer Pvt Ltd). An electronic apex locator (E-pex, Orikam Pvt Ltd) was used to determine the working length and was confirmed with radiovisiography (Fig 3). The coronal segment of the root canal was enlarged using Gates Glidden drill no.2 (Mani Pvt Ltd) followed by enlargement of the root canal to ISO size $40 \mathrm{~K}$-file (Dentsply Maillefer Pvt Ltd) till working length (WL $-18.5 \mathrm{~mm}$ ) and step back upto $60 \mathrm{~K}$-file (Dentsply Maillefer Pvt Ltd). During the biomechanical preparation 3\% Sodium hypochlorite (Modi Surgico Healthcare Pvt Ltd, Maharashtra, India), 17\% Ethylenediamine tetraacetic acid (PREVEST Den Pro) and 0.9\% Saline solution (Essential Dental Systems) were used for irrigation of the root canal. Paper points (Diadent Pvt Ltd) were used to dry the root canal and then master (gutta-percha) cone (Sybron endo) was selected (ISO No. 60, $2 \%$ taper) (Fig 4). The root canal was obturated with Zinc oxide eugenol sealer using lateral condensation obturation technique (Fig 5).

The post space was prepared and was extended beyond the fracture line with Peeso Reamer drills (No.3) (Mani Pvt Ltd) to receive the fiber reinforced post (Ivoclar Vivadent Pvt Ltd) (Fig 6). The fiber post was checked for the fit and size no 3 post was selected for cementation (Fig $7 \mathrm{a}, 7 \mathrm{~b}$ ). Multilink N-system (Ivoclar Vivadent Pvt Ltd) was used for fiber post cementation. Etching of the root canal was done using 37\% phosphoric acid (Aqua etch). Monobond N (Ivoclar Vivadent Pvt Ltd) was applied into the cavity and it was cured using curing light. The catalyst and base components of Multilink N (Ivoclar Vivadent Pvt Ltd) were mixed and applied following manufacturer's instructions. The mixed cement was carried into the root canal space with lentulo spiral (Dentsply Maillefer Pvt Ltd), the post was seated and excess material removed before light curing (Fig $8 \mathrm{a}, 8 \mathrm{~b}$ ). Core build up was done with composite (Fig 9). Tooth preparation was done i.r.t. 22. Upper and lower elastomeric impressions were made. Porcelain Fused to Metal crown cementation was done (Fig 10).
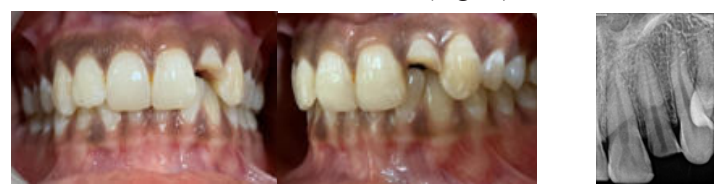

Fig 1a: Preoperative Fig 1b: Preoperative Fig 2: Preoperative Central view. - Lateral view. IOPA i.r.t 22. 


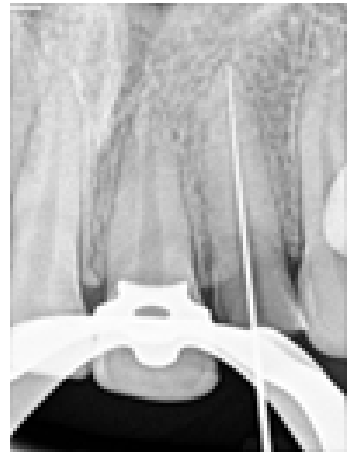

Fig 3: Working length determination i.r.t 22 .

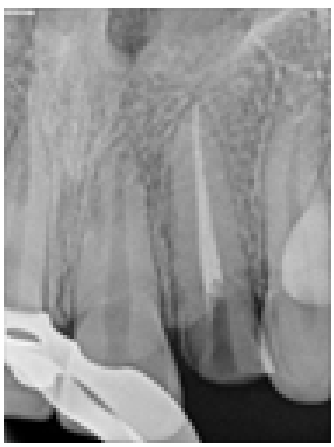

Fig 5: Obturation i.r.t 22.

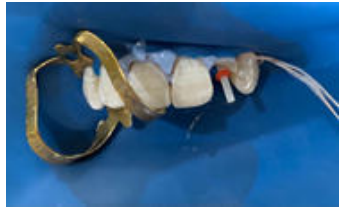

Fig 7a: Fiber post selection i.r.t 22.

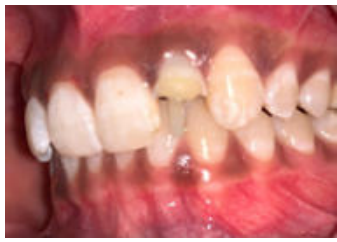

Fig 8a: Fiber post

cementation i.r.t 22.

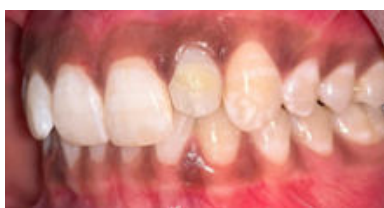

Fig 9: Core build up i.r.t 22.

\section{DISCUSSION:}

An attractive and pleasing smile with healthy teeth is an advantageous part of overall appearance and self-esteem. In the restoration of anterior teeth, depending on the patient's expectation, many factors should be considered. ${ }^{(6)}$

Traditionally cast posts have been used for a long time to restore complicated crown fracture. Depending on developments in adhesive dentistry resin-based fiber-reinforced posts have been used in the restoration of maxillary anterior teeth. ${ }^{(7)}$ Fiber resin posts show similar hardness to dentin and exhibits greater durability than the metal posts. Having elastic modulus similar to dentin strengthens the remaining tooth structure and increases resistance to tooth fracture. Because of these advantages, fiber post was used in this case to restore the fractured teeth. ${ }^{(8,9)}$ The potential problem regarding the original carbon fiber posts for post- restoration was the darkness of the material hampering the esthetics. More recent versions are white other types of fiber posts also are available, including quartz fiber, glass fiber, and silicon fiber posts. They are claimed to offer the same advantages as the carbon fiber posts, but with better esthetics. Because they are newer, most fiber posts are relatively radiolucent and have different radiographic appearance than traditional posts. ${ }^{(10)}$ Fiber posts are ready to use whereas more time, extra clinic and laboratory time are required for the metal post. ${ }^{(11)}$

The fiber-reinforced post offers clinical advantages with the ability of easy removal and being less traumatic. Like a cast or prefabricated post, fiber posts are not retrieved in one piece but are removed from the canal by drilling down directly through them. Much higher potential for causing allergic reactions and adverse biologic effects have been reported with metal post system. ${ }^{(12)}$

Olaide S Gbadebo et al. conducted a study to compare the clinical performance of metallic and glass fiber posts in the restoration of endodontically treated teeth and concluded that over a 6 month period, the rehabilitation of endodontically treated teeth using prefabricated glass fiber posts and metallic posts showed comparable clinical results. The clinical performance of the glass fiber post was slightly better than that of metallic post within the 6 months study period although this was not found to be statistically significant. However a long term review of the restorations will be required for further assessment. ${ }^{(13)}$

Roshan Uthappa, Deepika Mod et al. conducted a study to compare fiber post and metal post in the endodontically treated teeth restoration and concluded less chance of failure was seen with fiber post retained restored teeth than that of the metal post. ${ }^{(14)}$

\section{CONCLUSION:}

Excellent esthetic and functional results can be achieved with the use of a glass fiber-reinforced root canal post for the treatment of anterior traumatized teeth. Fiber post has better homogeneous tension distribution when loaded, than rigid metal or ceramic posts. Fiber reinforced posts also possess advantageous optical properties over metal or metal oxide post systems. Therefore, the use of fiber posts provides satisfying esthetic results and improved mechanical properties.

\section{REFERENCES:}

1. Geeta PS. Management of complex crown root fracture using fiber post- A case report. Endodontology.2014;26(1)

2. Mahajan N, Bansal S. Glass fiber-reinforced composite post and core used for restoration of traumatically fractured anterior teeth; Indian Journal of Oral Sciences. 2017, IP: 47.9.191.139

3. Garoushi S, Vallittu PK, Lassila LV. Continuous and short fiber reinforced composite in root post-core system of severely damaged incisors. Open Dent J 2009; 3:36-41.

4. Verma L, Passi S. Glass fiber-reinforced composite post and core used in decayed primary anterior teeth: A case report. Case Rep Dent 2011;864254.

5. Simone Grandin,Serena Sapio etal :Use of anatomic post and core for reconstructing and endodontically treated tooth: a case report; the journal of adhesive dentistry; 5(3),2003,243-247

6. Nandal SM, Nanda T. Fibre Post \& All Ceramic Crown- A Simple Approach to the Perfect Smile Sch. J. Dent. Sci., 2015; 2(3A):247-249.

7. C SK, Rao A, K S, G HR., Multidisciplinary Approach in Management of Fractured Central Incisor through Composite Plug Stabilization - A Case Report. J Int Oral Health 2013; 5:79-82.

8. Cormier CJ, Burns DR, Moon P. In vitro comparison of the fracture resistance an failure mode of fiber, ceramic and conventional post systems at various stages of restoration. JProsthodont 2001; 10:26-36.

9. Hayashi M, Sugeta A, Takahashi Y, Imazato S, Ebisu S. Static and fatigue fracture resistances of pulpless teeth restored with postcores. Dent Mater 2008; 24:1178-86.

10. Richard S. Schwartz, DDS, and James W. R Post Placement and Restoration of Endodontically Treated Teeth: ALiterature Review journal of endodontics. 2004.30(5).

11. Monticelli F, Grandini S, Goracci C, Ferrari M. Clinical behavior of translucent-fiber posts: A2-year prospective study. Int J Prosthodont 2003; 16:593-6.

12. Shashikala K, Sharma S. Clinical and radiological evaluation of cast metal and quartz fiber posts in endodontically restored teeth. Endodontology 2011;3:3746.

13. Gbadebo OS, Ajayi DM. Randomized clinical study comparing metallic and glass fibe post in restoration of endodontically treated teeth; Indian journal of dental research.2014; 25(1):58-63.

14. Uthappa R, Deepika Mod. Comparative evaluation of the metal post and fiber post in the restoration of the endodontically treated teeth; Journal of Dental Research. 2015.2(2):73-77. 